\title{
INSERCIÓN LABORAL EVALUADA POR EMPLEADORES
}

Andrés Correal Cuervo ${ }^{1}$, Ángela Carolina Bernal Álvarez², Juan Sebastián Cely Bottía ${ }^{3}$, Cristian Alejandro Aguilar Tovar ${ }^{4}$, Sandra Patricia Corredor Gamba ${ }^{5}$

\section{Resumen:}

Fomentar la empleabilidad supone disponer de una serie de competencias para gestionar la transición universidad-empleo. Así, desde la perspectiva de los empleadores, se señala que un profesional competente no es solamente aquel que sea capaz de desenvolverse en el mundo laboral, sino que debe poseer las competencias que le capaciten para vivir en sociedad. El objetivo del estudio fue evaluar la inserción laboral de los egresados de la Universidad de Boyacá desde la percepción de los empleadores. Se desarrolló a partir de una metodología cuantitativa y un estudio transversal con diseño descriptivo de muestreo probabilístico. Los resultados evidenciaron que el 66,1\% de los empleadores manifiestan tener un nivel de satisfacción "alto" respecto a la formación y desempeño de los profesionales, los cuales cuentan con habilidades acordes a las necesidades del entorno. Se concluye que son competentes en cualquier ámbito, como lo refiere el perfil de egreso de la Universidad de Boyacá.

Palabras clave: universidad, competencia profesional, calidad de la educación, formación (Unesco).

Historial del artículo:

Fecha de recibido: 26-10-2020 - Fecha de aceptado: 26-04-2021

(c) 2021. Fundación Universitaria Juan N. Corpas (FUJNC).

Artículo Open Access bajo la Licencia Creative Commons Atribución-

NoComercial-Compartirlgual 4.0 Internacional.

DOI: 10.26752/revistaparadigmassh.v3i1.511

\footnotetext{
${ }^{1}$ Ingeniero de sistemas. Magíster en Seguridad de la Información Empresarial. Candidato a doctor en Política y Gestión de la Educación. Rector de la Universidad de Boyacá. Universidad de Boyacá. Correo electrónico: sacorreal@uniboyaca.edu.co

${ }^{2}$ Administradora de Negocios Internacionales. Maestrante en Administración. Directora de la División de Egresados. Vicerrectoría de Desarrollo Institucional Universidad de Boyacá. Correo electrónico: angbernal@uniboyaca.edu.co

${ }^{3}$ Ingeniero industrial. Maestrante en Industria 4.0. Profesional de la División de Egresados.

Vicerrectoría de Desarrollo Institucional. Universidad de Boyacá. Correo electrónico:jscely@uniboyaca.edu.co

${ }^{4}$ Ingeniero industrial. Maestrante en Gestión Humana y Desarrollo Organizacional. Correo electrónico: caaguilar@uniboyaca.edu.co ${ }^{5}$ Profesional en Terapia Respiratoria. Magíster en Actividad Física: Entrenamiento y Gestión Deportiva. Profesional de la División de Egresados. Vicerrectoría de Desarrollo Institucional. Universidad de Boyacá. Autor para correspondencia.

Correo electrónico: sancorredor@uniboyaca.edu.co
} 


\title{
EMPLOYER ASSESSED JOB PLACEMENT
}

\begin{abstract}
:
Promoting employability means having a series of competencies to manage the transition from university to employment; from the perspective of employers, it is pointed out that a competent professional is not only one who is capable of performing in the labor world, but one who has the competencies that enable him/her to live in society. The objective of the study was to evaluate the labor insertion of the graduates of the University of Boyacá from the perception of the employers. It was developed from a qualitative methodology, a cross-sectional study with a descriptive design of probability sampling. The results showed that $66.1 \%$ of the employers stated that they had a "high" level of satisfaction with the training and performance of the professionals, who had skills relevant to the needs of the environment. Concluding that they are competent in any field as referred to the profile of graduation from the University of Boyacá.
\end{abstract}

Keywords: universities, occupational qualifications, educational quality, training (Unesco). 


\section{Introducción}

Para analizar los procesos de inserción en el mercado de los profesionales egresados de instituciones de educación superior es necesario, en primera instancia, describir los rasgos del mercado con el que se van a encontrar una vez finalizados sus estudios. Estas características van a condicionar la trayectoria profesional, por lo que es pertinente pasar a analizar las pautas que determinan la transición del sistema educativo al laboral.

Hasta el momento, es posible deducir que las grandes condicionantes de la inserción laboral en el nuevo mercado al que se enfrentan los profesionales, a nivel general, una vez que finalizan sus estudios, se centran en la flexibilidad laboral, la cual ha originado movilidad en el empleo, lo que incrementa la tasa de contrataciones, pero disminuye la estabilidad de este. Así mismo, el desempleo, junto con la generalización de los niveles superiores de educación a los que acceden los profesionales, ha traído consigo un incremento de la competitividad por los puestos de trabajo, lo que generaliza el fenómeno de la sobrecualificación y evidencia una falta de ajuste entre la formación ofrecida en los diferentes entornos formativos y las exigencias del mercado sociolaboral.

Un nuevo concepto a tener en cuenta a la hora de analizar la inserción laboral de los profesionales es el de empleabilidad. Según García \& Cárdenas (2017), la empleabilidad es el grado de tracción que un individuo tiene para el mercado laboral a lo largo del tiempo. Se puede decir que existe un cierto consenso respecto a que la empleabilidad requiere una inclusión en el proceso formativo que recoja la evidencia e investigación especializada al respecto (Burke et al., 2017); así mismo, se destaca la relevancia de la inserción curricular, además de la extracurricular (Artess et al., 2017), lo que se materializa en la valoración que genera el empleador o la sociedad en su conjunto.
La adopción del concepto de empleabilidad ha supuesto una notable transformación en la aproximación que, en el mundo laboral, se realiza sobre la formación impartida. Con ello, la formación se percibe como una herramienta inicial cuyo objetivo principal se centra en la adquisición de los conocimientos, habilidades y destrezas que serán necesarias para desarrollar en una actividad profesional. También se ha contemplado como una actividad continua que se desarrolla en la trayectoria profesional y laboral. Así, el grado de vinculación entre los individuos y el empleo (estable, de calidad y a largo plazo) depende de una serie de cualidades, capacidades, motivaciones o intereses necesarios y eficientes. En este sentido, estas transformaciones hacen necesario disponer de competencias asociadas a una capacidad de cambio que favorezcan la empleabilidad (Rico, 2003). Desde esta perspectiva, se habla de empleabilidad como el desarrollo de habilidades para la gestión de la carrera, pues se destacan planteamientos orientados a crear vínculos entre la actividad formativa y la vida laboral, que prepara para el desempeño y estimula el desarrollo de la carrera (McCarthy, 2016).

Las competencias se convierten, por tanto, en elementos clave en la comprensión del concepto de empleabilidad y, al mismo tiempo, del perfil de egreso y profesional, ya que definen la identidad profesional de los sujetos, que, con una titulación académica determinada, Ilevan a cabo una labor categórica acorde a su disciplina. Empero, el principal ejemplo acerca del efecto de invisibilización de dichos aportes se evidencia en el uso simplista y recurrente de la empleabilidad como sinónimo de empleo (Burke et al., 2017).

El impacto de la formación universitaria en las posibilidades de conseguir empleo depende en gran medida de la postulación de cada disciplina o profesión, lo que, a su vez, está determinado tanto por la oferta como por la demanda de profesionales, es decir, por el equilibrio exis- 
tente para cada profesión entre el sistema educativo y el mundo laboral (Rothwell $\&$ Rothwell, 2017).

De acuerdo con lo referido por Latiesa et al. (2001), el problema de encontrar un empleo que sea adecuado a las cualificaciones de los profesionales es propio de las sociedades industrializadas; lo anterior, al tener en cuenta que los profesionales acceden a trabajos más estables, mejor remunerados y más adecuados a sus competencias; así mismo, la discriminación por razón de género es menor que en el caso de otros trabajadores menos cualificados.

En consecución, las competencias, tanto genéricas como específicas, posibilitarán una mayor vinculación entre la educación impartida en la institución y el empleo, puesto que se formarán profesionales competentes y adaptables a nuevos retos. Al mismo tiempo, se pone en evidencia la necesaria y compleja integración en los planes curriculares de la formación y la evaluación basada en competencias. Estos aspectos, según Villa \& Villa (2007), deben integrar variables relacionadas con las actitudes y valores, conocimiento, habilidades intelectuales, técnicas y destrezas, normas y procedimientos que diferencian la actuación y el comportamiento en el correcto desempeño académico actual y profesional a futuro.

Al tener en cuenta este contexto, la gestión por competencias laborales en las empresas obedece en parte a la necesidad de acortar la distancia entre esfuerzo de formación y resultado efectivo (Mertens, 2001). El concepto de competencia laboral se utilizó inicialmente en los países industrializados, a partir de la necesidad de formar profesionales capaces de responder a los cambios tecnológicos, organizacionales y, en general, a la demanda de un nuevo mercado laboral. A su vez, en los países en desarrollo, su aplicabilidad ha estado asociada al mejoramiento de los sistemas de formación para lograr un mayor equilibrio entre las necesidades de las personas, empresas y sociedades en general.
Cabe mencionar a partir de la Constitución Política de Colombia de 1991, la educación nacional ha sufrido replanteamientos normativos, lo que ha permitido establecer nuevas alternativas para incluir en la política educativa nacional. A este respecto, se han generado discusiones y concertaciones sobre aspectos filosóficos y pedagógicos en la educación acordes a los tiempos actuales. Con ellos, lo que se pretende es generar una reestructuración y revolución educativa que impulse un nuevo comportamiento cultural, que además permita la maximización de las capacidades intelectuales y organizativas (Misión de Ciencia, Educación y Desarrollo, 1994). En este sentido, se afirma que no solo es necesario poseer el conocimiento, sino asegurar competencias que preparen al futuro profesional.

Es allí cuando la institución de educación superior entra a desempeñar un papel fundamental en su calidad de formador de conocimiento, pues debe cumplir estas funciones de manera tal que promueva la competitividad, a la vez que garantice la inserción y movilidad laboral de los profesionales. Lo anterior implica alinear las estrategias y recursos pedagógicos de las instituciones con las condiciones y necesidades del contexto, de manera que los egresados y la sociedad en general cuenten con las herramientas e instrumentos intelectuales suficientes e idóneos para desenvolverse en el medio laboral. Para el sector educativo, esto implica ampliar sus perspectivas con información sobre la dinámica y tendencias de su entorno, acercarse al mundo productivo para establecer alianzas, contribuir con una educación pertinente y de buena calidad, y ser parte activa y fundamental de la estrategia de competitividad y productividad (García et al., 2019).

En este orden de ideas, la universidad, en su proceso de formación académica y en sus disciplinas profesionales, tiene unos objetivos claros con los egresados que se vinculan en el contexto local, regional, nacional e internacional, el cual debe responder a los retos de un 
mundo laboral cambiante e influyente (Tomlinson, 2017). En general, la institución es la responsable de incurrir en variables asociadas a la generación del capital humano, en el cual se ve la necesidad de contar con personas que posean unos modelos mentales orientados a los resultados, calidad, aprendizaje continuo, creatividad, afrontamiento de la incertidumbre. Lo anterior es posible con la adquisición de competencias generales y específicas, que les permitan emplear sus recursos cognitivos para trabajar, producir conocimientos, desarrollar habilidades y destrezas específicas bajo valores y principios éticos, aspectos centrales de los debates en torno a la relación entre la educación superior y el mundo laboral (Orellana, 2018).

En particular, los egresados de la Universidad de Boyacá son actores fundamentales de los procesos académicos, investigativos y de proyección social, funciones sustantivas institucionales. Para el cumplimiento de ello, se ha fortalecido su compromiso al dar respuesta de manera oportuna, eficiente y con calidad a los requerimientos de una sociedad que permanece en constante cambio, al tener en cuenta factores como los avances de la ciencia y la tecnología. La institución concibe el perfil de ingreso y de egreso como un sistema dinámico de interacción, fundamentado en la formación integral, en la que encajan cada uno de los elementos del proceso de formación que involucra el desarrollo de competencias, saberes, valores, elementos disciplinares, investigativos, tecnológicos y de proyección social. Esta interacción permitirá la transformación del aspirante en un profesional altamente competente.

En este sentido, la calidad como aspecto fundamental de los sistemas e instituciones de educación universitaria reflejan el deseo de los profesionales de ubicarse laboralmente, en que "ser competitivo es una condición que determina el crecimiento y la permanencia" (Batista \& Guacari, 2019), lo que reclama la relevancia de las instituciones de educación superior para formar profesionales idóneos. El desempeño de los egresados no solo preocupa a los participantes en el proceso educativo (directivos, docentes y estudiantes), sino también a los empresarios y empleadores que consideran a las instituciones universitarias como centros de capacitación de profesionales de alto nivel y de producción de conocimientos esenciales para mantener el desempeño profesional y laboral (Zarta Barrios, 2017; Corredor et al., 2020).

Los procesos de autoevaluación por programas e institucional contemplan como parte de uno de sus factores el desempeño profesional de sus egresados. De acuerdo con las políticas del Consejo Nacional de Acreditación (CNA), el seguimiento a los egresados se constituye en uno de los factores que requiere del análisis en el proceso de cualificación permanente en el interior de los programas académicos y de la institución en general. Dicho análisis se debe construir a partir de la información suministrada por los egresados y como resultado de la comparación entre la formación recibida y la realidad laboral (Garzón, 2018). De acuerdo con los lineamientos de acreditación, un programa de alta calidad se reconoce por medio del desempeño profesional y laboral de los egresados y del impacto que estos tienen en los procesos de desarrollo académico, investigativo, social, cultural y económico, evaluado directamente por los empleadores.

De esta manera, el presente estudio evaluó la inserción laboral de los egresados de la Universidad de Boyacá desde la percepción de los empleadores, con el fin de crear un mecanismo que le permita a la institución conocer la calidad de la formación académica impartida, para así cumplir con las exigencias y necesidades del mercado laboral actual.

\section{Metodología}

Se realizó un estudio de metodología cuantitativo de tipo transversal con diseño descriptivo. Para la selección de la muestra, se consideraron empresas de orden local, regional, nacional e 
internacional que tuvieran vinculados profesionales egresados de la Universidad de Boyacá. Para la construcción del instrumento de recolección de información, se tomó como hoja de ruta el formulario dispuesto para tal fin por el Observatorio Laboral para la Educación (OLE). No obstante, se modificaron algunas secciones de dicho cuestionario para incluir aspectos relevantes del modelo pedagógico de la institución y de esta forma garantizar que la información obtenida permitiera evaluar la pertinencia de los contenidos académicos y el desempeño de los profesionales egresados.

La muestra se tomó de empleadores de 188 empresas y la información fue recogida entre los meses de marzo a mayo del 2018, a través del diligenciamiento de la encuesta de manera física y digital, según la disponibilidad de los empleadores participantes en el estudio.

El procesamiento de los datos se realizó con el paquete estadístico SPSS ${ }^{\circledR}$ versión 25.0, que realizó un análisis univariado descriptivo y calculó frecuencias, porcentajes, medidas de tendencia central e intervalos de confianza. Se cuidaron los aspectos éticos en relación con el consentimiento informado y la privacidad de la información. Finalmente, se contó con el aval institucional por parte del comité de bioética a nivel institucional y los gastos fueron cubiertos por la Universidad de Boyacá.

Tabla 1. Niveles de satisfacción de los empleadores encuestados con la formación y el desempeño del egresado

\begin{tabular}{|l|c|c|c|c|}
\hline \multicolumn{1}{|c|}{ Variable } & $\mathrm{n}^{\circ}$ & $\%$ & \multicolumn{2}{c|}{ Intervalo de Confianza } \\
\hline $\begin{array}{l}\text { Formación y necesidades } \\
\text { de la empresa }\end{array}$ & 101 & $54 \%$ & 44.2 & 63.7 \\
Muy alto & 85 & $45 \%$ & 34.4 & 55.5 \\
Aceptable & 1 & $1.0 \%$ & 1.0 & 6.0 \\
Bajo & 49 & $26 \%$ & 13.7 & 38.2 \\
Desempeñite & $\begin{array}{c}\text { Límite } \\
\text { Inferior }\end{array}$ \\
Muy alto & 78 & $42 \%$ & 31.0 & 52.9 \\
Alto & 60 & $32 \%$ & 20.1 & 43.8 \\
Aceptable & & & & \\
\hline
\end{tabular}

Fuente: elaboración propia. 


\section{Resultados}

Niveles de satisfacción con respecto al desempeño y a la formación de los egresados

Los empleadores encuestados manifestaron tener un nivel de satisfacción generalmente positivo con la formación de los egresados, lo que se ve reflejado en el 54\% (IC 95\%, 44.2-63.7) de las empresas objeto de estudio, que expresaron sentir un nivel "Muy alto" de satisfacción, el cual fue el nivel más elegido por los encuestados. Como segundo nivel de satisfacción, se encuentra el "Aceptable", con una diferencia porcentual del 9\% sobre el del nivel "Muy alto". Así mismo, se presenta una pequeña propor- ción 1\% (IC 95\%, 1.0-6.0) de los empleadores encuestados que indican tener un nivel "Bajo" de satisfacción con la formación de los egresados. En un caso particular, hubo quienes no contaban con una tendencia definida.

La satisfacción general con el desempeño de los egresados es favorable, pues los empleadores perciben un desempeño laboral adecuado a las necesidades y exigencias propias de su cargo, en muchos casos con creces. Sin embargo, existen grupos de empleadores que consideran que el desempeño, si bien cumple con sus requerimientos básicos, palidece en aspectos importantes para ellos. Con lo anterior se pueden

Figura 1. Nivel de satisfacción con el desempeño laboral de los egresados por facultad

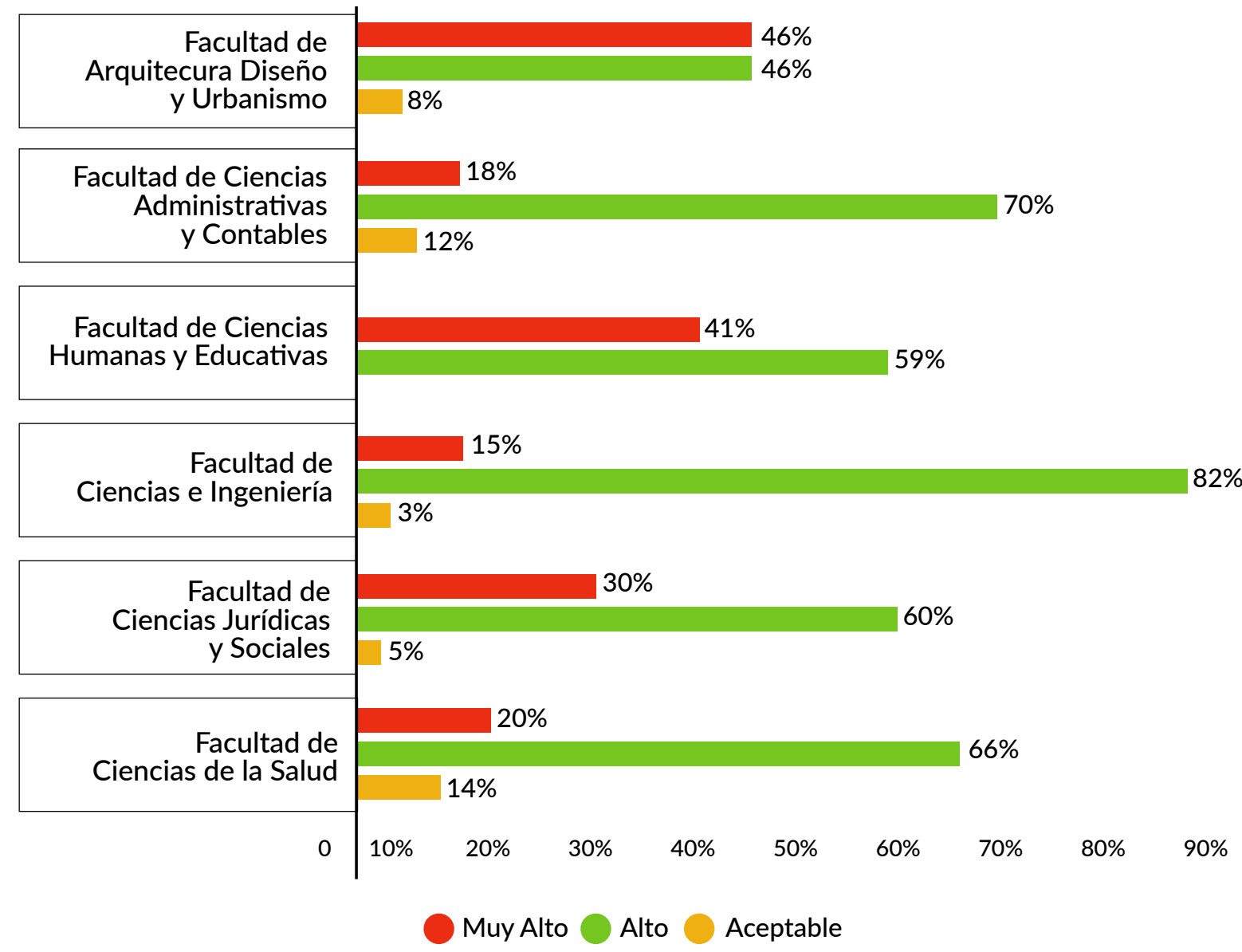

Fuente: elaboración propia. 
justificar los niveles de satisfacción positivos "Alto" con un $42 \%$ (IC 95\%, 31.0-52.9), seguido de "Muy alto" con un 26\% (IC 95\%, 13.7).

La satisfacción general con el desempeño de los egresados es "Alta", con el $66 \%$ del total de encuestados, y se refleja en las proporciones de cada facultad. Como datos notables, la Facultad de Arquitectura, Diseño y Urbanismo presenta proporciones iguales en las calificaciones "Muy alta" y "Alta" en el desempeño laboral, mientras que ninguno de los empleadores de la Facultad de Ciencias Humanas y Educativas calificaron como "Aceptable" su satisfacción con el desempeño de los egresados. Esto también contrasta con la Facultad de Ciencias de la Salud, en la que dicha calificación obtuvo mayor representación que en las demás facultades.

\section{Competencias genéricas}

La Universidad de Boyacá en el desarrollo de su proyecto estratégico y según su filosofía visional y misional ha incorporado en la gestión académica su propia versión de la formación basada en competencias, con el fin de auspiciar el desarrollo integral del educando como elemento sustancial de la responsabilidad social de formar profesionales. Los egresados, además de responder a las exigencias del entorno en materia del saber y saber hacer, deben desarrollar las condiciones personales para saber ser y convivir como ciudadanos integrales y seres humanos trascendentes, capaces de transformar y conducir la comunidad hacia escenarios de libertad, justicia, igualdad, convivencia pacífica y desarrollo socioeconómico sostenible. Con ello, podrá demostrar la capacidad para manejar los contextos propios de su profesión y transversalmente competentes para propiciar la integración y el diálogo de saberes y sentires como una forma de acercar objetivamente la ciencia al servicio de la sociedad( Universidad de Boyacá, 2016).

Para el presente estudio, se solicitó a los empleadores encuestados (jefes directos) hacer una evaluación de las competencias generales de la universidad, con la calificación de cada elemento que compone la competencia en un nivel de importancia para las empresas y un nivel del logro que evidencian los egresados (de 1 a 4). Para dicha evaluación, se tomó como referencia el formulario establecido por el Observatorio Laboral para la Educación (OLE) y se reorganizó con el fin de visualizar las competencias establecidas en el modelo pedagógico institucional.

El análisis de las competencias generales, al momento de su evaluación, consideró la importancia que da el empleador a cada elemento y el grado de cumplimiento que observa en el desempeño del graduado. Lo anterior, para determinar la brecha entre ambas calificaciones y así identificar las competencias consideradas fortalezas o debilidades en el perfil de egreso de la Universidad de Boyacá. La calificación mencionada se realiza del 1 al 4, siendo 1 la menor calificación y 4 la mayor. 


\begin{tabular}{|c|c|c|}
\hline \multicolumn{3}{|l|}{ Competencia } \\
\hline Ético-ciudadana & Importancia & Logro \\
\hline Formación en valores y principios éticos. & 4.0 & 3.9 \\
\hline \multicolumn{3}{|l|}{ Liderazgo y manejo del cambio. } \\
\hline Asume responsabilidades y toma decisiones. & 3.9 & 3.6 \\
\hline $\begin{array}{l}\text { Planifica y utiliza el tiempo de manera efectiva para } \\
\text { el logro de los objetivos planteados. }\end{array}$ & 3.9 & 3.6 \\
\hline $\begin{array}{l}\text { Trabaja en equipo para alcanzar una meta en } \\
\text { común. }\end{array}$ & 3.9 & 3.7 \\
\hline Trabaja de forma independiente. & 3.8 & 3.5 \\
\hline Identifica, plantea y resuelve problemas. & 3.8 & 3.5 \\
\hline $\begin{array}{l}\text { Se adapta a los cambios y resuelve imponderables } \\
\text { fácilmente. }\end{array}$ & 3.8 & 3.5 \\
\hline $\begin{array}{l}\text { Posee gran capacidad para trabajar } \\
\text { bajo presión. }\end{array}$ & 3.7 & 3.5 \\
\hline \multicolumn{3}{|l|}{$\begin{array}{l}\text { Investigativa y de procesamiento de la } \\
\text { información }\end{array}$} \\
\hline $\begin{array}{l}\text { Utiliza herramientas informáticas básicas } \\
\text { (procesadores de texto, hojas de cálculo, etc). }\end{array}$ & 3.7 & 3.6 \\
\hline $\begin{array}{l}\text { Utiliza herramientas informáticas especializadas } \\
\text { (paquetes estadísticos, programas de diseño, etc). }\end{array}$ & 3.3. & 3.0 \\
\hline $\begin{array}{l}\text { Disposición para aprender y } \\
\text { mantenerse actualizado. }\end{array}$ & 3.9 & 3.6 \\
\hline $\begin{array}{l}\text { Formula y gestiona proyectos procedentes de } \\
\text { fuentes y áreas diversas. }\end{array}$ & 3.4 & 3.0 \\
\hline Abstrae, analiza y sintetiza de manera adecuada. & 3.7 & 3.4 \\
\hline \multicolumn{3}{|l|}{ Comunicativa } \\
\hline $\begin{array}{l}\text { Comunica pensamientos, ideas e información de } \\
\text { forma oral para que los demás entiendan. }\end{array}$ & 3.8 & 3.6 \\
\hline $\begin{array}{l}\text { Comunica pensamientos, ideas e información por } \\
\text { escrito. }\end{array}$ & 3.7 & 3.3 \\
\hline Habla y escribe en un idioma extranjero. & 2.6 & 2.3 \\
\hline $\begin{array}{l}\text { Presenta y sustenta adecuadamente en público } \\
\text { informes e ideas. }\end{array}$ & 3.7 & 3.4 \\
\hline
\end{tabular}

Fuente. elaboración propia. 


\section{Competencia ético-ciudadana}

Al considerar que esta competencia está comprendida por un elemento, se observa que el promedio del puntaje asignado por los empleadores en cuanto a la importancia y el logro no difiere en gran medida, pues el promedio de calificación que dio el empleador para la importancia de este elemento fue de 4.0 y el promedio del logro que evidencia el egresado según la opinión de los empleadores encuestados es de 3.9.

\section{Competencia de liderazgo y manejo del cambio}

En este caso, los elementos que mayor importancia tienen para los empleadores de los egresados de la Universidad de Boyacá son: "asume responsabilidades y toma decisiones", "planifica y utiliza el tiempo de manera efectiva para el logro de los objetivos planteados" y "trabaja en equipo para alcanzar una meta común". Así mismo, el elemento que mayor puntaje tuvo por consideración del logro de los egresados según la opinión de los empleadores es el de "trabaja en equipo para alcanzar una meta en común", que no difiere de la calificación considerada por los empleadores para la importancia.

\section{Competencia investigativa y de procesa- miento de la información}

Como se observa en la tabla 2, en promedio, los empleadores encuestados consideraron que el elemento de la competencia que mayor importancia tiene según el puntaje es la "disposición para aprender y mantenerse actualizado", lo que no difiere en gran medida del logro de los egresados graduados alcanzado para dicho aspecto.

Así mismo, en los elementos se encuentran: "utiliza herramientas informáticas especializadas (paquetes estadísticos, programas de diseño, etc.)" y "formula y gestiona proyectos procedentes de fuentes y áreas diversas"; hay un puntaje promedio de 3.0, al ser calificados como los más bajos de esta competencia, aunque no difiere de la calificación en importancia referida por los empleadores.

\section{Competencia comunicativa}

El elemento denominado: "comunica pensamientos, ideas e información de forma oral para que los demás entiendan" tuvo el puntaje más alto en la importancia que tiene para los empleadores.

Con respecto al elemento de "habla y escribe en un idioma extranjero", se observa que los empleadores encuestados consideraron el puntaje más bajo tanto para la importancia como para el logro que evidencian los egresados. Este resultado es la calificación más baja en referencia a los elementos de todas las competencias anteriormente presentadas, lo que deja ver una falencia que debe ser planteada en los planes de mejora a nivel académico.

\section{Brecha entre importancia y logro de las com- petencias genéricas}

Para la generación de las brechas, se calculó la diferencia de medias $(x)$ entre la importancia y el logro que evidencian los egresados graduados, ante los elementos de las competencias generales institucionales, según opinión de empleadores. Con base en lo anterior, la tabla 3 muestra los rangos establecidos para definir el tipo de brecha (baja, media o alta) y el tiempo para generar acciones de mejora. Lo anterior tiene como base el estudio que llevó a cabo la Agencia Nacional de Evaluación de la Calidad y Acreditación (ANECA), en colaboración con el Centro de Estudios en Gestión de la Educación Superior de la Universidad Politécnica de Valencia (CEGES), desarrollada en Madrid (España) en el año 2008. 
Tabla 3. Definición de brechas de las competencias generales

\begin{tabular}{|c|c|c|c|}
\hline Tipo de brecha & Rango de brecha (x) & Rango de brecha (\%) & $\begin{array}{c}\text { Requerimiento de } \\
\text { acciones }\end{array}$ \\
\hline Baja & (de 0.0 a 0.9) & (del 0\% al 33\%) & $\begin{array}{c}\text { Acción de mejora a } \\
\text { largo plazo } \\
\text { Media }\end{array}$ \\
Alta & (de 1.0 a 1.9) & (del 34\% al 66\%) & $\begin{array}{c}\text { Acción de mejora a } \\
\text { mediano plazo }\end{array}$ \\
(de 2.0 a 3.0) & (del 67\% al 100\%) & $\begin{array}{c}\text { Acción de mejora a } \\
\text { corto plazo }\end{array}$ \\
\hline
\end{tabular}

Fuente: elaboración propia.

Tabla 4. Brecha entre importancia y logro de las competencias genéricas

\begin{tabular}{|c|c|}
\hline Competencia & $\begin{array}{l}\text { Brecha entre } \\
\text { importancia y logro }\end{array}$ \\
\hline Ético-ciudadana & Importancia \\
\hline Formación en valores y principios éticos & $3.3 \%$ \\
\hline \multicolumn{2}{|l|}{ Liderazgo y manejo del cambio } \\
\hline Asume responsabilidades y toma decisiones. & $10.86 \%$ \\
\hline $\begin{array}{l}\text { Planifica y utiliza el tiempo de manera efectiva para el } \\
\text { logro de los objetivos planteados. }\end{array}$ & $11.13 \%$ \\
\hline $\begin{array}{l}\text { Trabaja en equipo para alcanzar una meta en común } \\
\text { Trabaja de forma independiente. }\end{array}$ & $5.67 \%$ \\
\hline Identifica, plantea y resuelve problemas. & $7.79 \%$ \\
\hline $\begin{array}{l}\text { Se adapta a los cambios y resuelve imponderables } \\
\text { fácilmente. }\end{array}$ & $10.35 \%$ \\
\hline Posee gran capacidad para trabajar bajo presión. & $12.31 \%$ \\
\hline \multicolumn{2}{|l|}{ Investigativa y de procesamiento de la información } \\
\hline $\begin{array}{l}\text { Utiliza herramientas informáticas básicas } \\
\text { (procesadores de texto, hojas de cálculo, etc). }\end{array}$ & $6.92 \%$ \\
\hline $\begin{array}{l}\text { Utiliza herramientas informáticas especializadas } \\
\text { (paquetes estadísticos, programas de diseño, etc). }\end{array}$ & $0.01 \%$ \\
\hline Disposición para aprender y mantenerse actualizado. & $5.12 \%$ \\
\hline $\begin{array}{l}\text { Formula y gestiona proyectos procedentes de fuentes } \\
\text { y áreas diversas. }\end{array}$ & $5.48 \%$ \\
\hline Abstrae, analiza y sintetiza de manera adecuada. & $15.32 \%$ \\
\hline \multicolumn{2}{|l|}{ Comunicativa } \\
\hline $\begin{array}{l}\text { Comunica pensamientos, ideas e información de forma } \\
\text { oral para que los demás entiendan. }\end{array}$ & $11.11 \%$ \\
\hline $\begin{array}{l}\text { Comunica pensamientos, ideas e información } \\
\text { por escrito. }\end{array}$ & $7.18 \%$ \\
\hline Habla y escribe en un idioma extranjero. & $\begin{array}{l}14.3 \% \\
8.8 \%\end{array}$ \\
\hline $\begin{array}{l}\text { Presenta y sustenta adecuadamente en público } \\
\text { informes e ideas. }\end{array}$ & $11.18 \%$ \\
\hline
\end{tabular}

Fuente: elbaración propia. 
La competencia ético-ciudadana presentada en la tabla 4 se compone de un único elemento denominado "formación en valores y principios éticos", el cual presenta una brecha de importancia/logro baja, lo que plantea la necesidad de acciones de mejora a largo plazo. Estos resultados van encaminados a mantener el contenido curricular de la asignatura de ética como una actividad académica transversal, de tal manera que los egresados sean evaluados por sus empleadores como unos profesionales íntegros y que contribuyen a la convivencia ciudadana.

Respecto a la competencia de liderazgo y afrontamiento al cambio, se señala que todos los elementos evaluados representan una acción de mejora a largo plazo, puesto que la brecha no supera el porcentaje del 33\%.

Así pues, el elemento denominado "se adapta a los cambios y resuelve imponderables fácilmente" presenta la brecha más alta, lo que plantea una acción de mejora a largo plazo y apunta a la necesidad de reforzar los syllabus o programas de asignaturas que permitan que los futuros egresados desarrollen habilidades para plantear soluciones a circunstancias imprevisibles. Lo anterior tiene relación con la brecha que respecta al elemento "identifica, plantea y resuelve problemas".

En el elemento "asume responsabilidades y toma decisiones", se observa una brecha que sugiere una acción a largo plazo, seguida del componente denominado "planifica y usa el tiempo de manera efectiva para el logro de los objetivos planteados".

Es de resaltar que, para el elemento llamado "trabaja en equipo para alcanzar una meta común", la brecha entre la importancia y el logro fue la menor evaluada, resultado que propone una acción de mejora a largo plazo. Es recomendable realizar una revisión de los conteni- dos programáticos que permiten desarrollar esta destreza en los futuros egresados de la institución.

La competencia comunicativa presenta una brecha que sugiere acciones de mejora a largo plazo, como se aprecia en la tabla 4. La brecha más alta que recomienda acciones a mediano plazo se presenta en el elemento que evaluaba la comunicación de pensamientos, ideas e información por escrito y sugiere la revisión de los contenidos programáticos de asignaturas transversales referentes a expresión escrita. De igual forma, el elemento denominado "presenta y sustenta adecuadamente en público informes e ideas" propone hacer una revisión de las asignaturas que desarrollen actividades referentes a la expresión de pensamientos e ideas en público. En coherencia con este resultado, se observa la brecha del elemento de la comunicación de pensamientos, ideas e información de forma oral para que los demás entiendan, la cual atribuye el resultado a las sugerencias anteriormente expuestas a largo plazo.

La apreciación de los empleadores con respecto al elemento denominado "habla y escribe en un idioma extranjero" presentó una brecha que indica plantear estrategias de mejora a largo plazo, de tal manera que los futuros graduados puedan responder a las necesidades del mercado laboral.

La competencia investigativa y de procesamiento de la información cuenta con cinco elementos, presentados en la tabla 4, de los cuales "formula y gestiona proyectos procedentes de fuentes y áreas diversas" presenta una brecha alta. Los elementos denominados "utiliza herramientas informáticas especializadas", "tiene la disposición de aprender y mantenerse actualizado" y "abstrae, analiza y sintetiza de manera adecuada" advierten acciones de mejora a mediano plazo. 
Con un porcentaje menor, el uso de herramientas informáticas básicas señala la brecha más baja de la competencia, a la que se le atribuye la necesidad identificada por la universidad de incorporar en los currículos de todos los programas la asignatura de informática básica.

\section{Discusión}

Las dificultades que se presentan en el mercado laboral constituyen una tendencia generalizada a nivel mundial y se puede decir que desde hace varios años se han implementado políticas de flexibilización laboral (Solé et al., 2018). El egresado de la Universidad de Boyacá debe competir de forma decidida con otros profesionales en sus propias áreas de formación, así como con otros profesionales, si bien los empleadores refieren estar satisfechos con el desempeño de los profesionales egresados de la universidad.

El seguimiento a los egresados para las instituciones de educación superior se constituye en una estrategia de evaluación y autorregulación. Así, a través de la implementación, desarrollo y ejecución de estos estudios se conoce información relevante que puede conducir a formular políticas de mejoramiento o de direccionamiento institucional. Las instituciones de educación superior deben, entre sus responsabilidades, ofrecer educación de calidad y que esta beneficie a los estudiantes, egresados, organizaciones y, en general, a la sociedad (Garzón, 2018).

La información proporcionada por los empleadores confirma la tergiversación y el desconocimiento con que tiende a usarse el término competencia (Lévy, 2003) y la falta de consenso existente en torno a este (Tardif, 2008). Para la Comisión Europea (2004), el concepto de competencia indica "una combinación de destrezas, conocimientos, aptitudes y actitudes, así como a la inclusión de la disposición para aprender, además de saber cómo" (p. 5). Este concepto, asumido por gran parte de la comunidad aca- démica, pone el énfasis en la articulación de estos elementos y en "un saber actuar complejo que se apoya sobre la movilización y utilización eficaz de una variedad de recursos didácticos, académicos, investigativos, entre otros" (Tardif, 2008, p.3). Sin embargo, los empleadores del presente estudio le atribuyen un significado que está más ligado a las capacidades, habilidades, actitudes y aptitudes de los egresados, al ser evaluados en altos niveles, acorde a las necesidades de las labores asignadas.

La calificación del desempeño de los profesionales según la opinión de los empleadores refleja un buen nivel en las competencias, no obstante, fue posible identificar algunas áreas de oportunidad vinculadas en mayor medida al contexto organizacional, como lo es la búsqueda de mejora del servicio, el manejo de conflictos, la disposición y enfrentamiento al cambio, la creatividad, así como la capacidad crítica y autocrítica (Guartán et al., 2019). En concordancia con esto y de acuerdo a los resultados obtenidos en la investigación, en un estudio amplio realizado por la Universidad de Valencia en 2007, se encontró que las características más valoradas por los empleadores correspondían a las competencias generales en las que se destacaban la capacidad para la solución de problemas, el asumir responsabilidades, el trabajo en equipo, la planificación y la gestión de recursos (Peiro et al., 2009).

Los empleadores valoran, principalmente, las competencias generales. En este sentido, se aprecia cierta similitud con los resultados de la investigación realizada por Freire et al. (2011) que confirman a las competencias generales como las más valoradas en el mercado laboral, las cuales son igualmente subrayadas en las investigaciones realizadas por Palmer et al. (2009), Rodríguez et al. (2012), Martínez et al. (2018) y Jackson (2012). 
En general, los empleadores tienden a dar mucha importancia a la competencia del hacer y saber hacer, y en más bajo porcentaje a las competencias del saber. Sin embargo, es importante destacar que en ningún caso mencionan la relación teórico-práctica, aunque en la universidad esta conexión se considera clave para garantizar un correcto desarrollo de las competencias. Situaciones como estas son las que llevan a algunos autores a exigir un mejor engranaje entre la formación impartida en las instituciones de educación superior y el mercado laboral (Cifuentes, 2017; Jackson, 2012), tal como se evidencia en los resultados del estudio.

En opinión de los empleadores, el desarrollo de competencias en la universidad requiere de un trabajo coordinado entre administración, universidad y empresas, como también subraya Jackson (2012). Esta tarea conlleva cambios estructurales que afectan a la relación que mantiene la administración educativa con las universidades y a la de estas con las empresas. Los cambios estructurales que se proponen responden a las decisiones que deben adoptarse y las mejoras en diferentes momentos: antes de empezar los estudios universitarios, durante la realización de estos y en la formación continua y en la evaluación posgradual.

Aunque es conocido que las instituciones de educación superior se mueven a un ritmo más acelerado de lo que puede realizarse en la actualización de los programas y planes de estudio, los resultados confirman la validez de los estudios de opinión con empleadores, estos como un medio idóneo para evaluar las necesidades y requerimientos que deben trabajarse en las instituciones educativas. Como se ha señalado, los estudios de opinión de empleadores, además de permitir conocer y medir el grado de satisfacción del conjunto de empleadores acerca del desempeño laboral de los egresados universitarios, permiten de manera implícita evaluar el currículum, el perfil profesional y el grado de desarrollo y aplicación de las competencias genéricas y específicas de los egresado (Del Socorro, 2017).

Con respecto al grado de satisfacción del empleador con relación al nivel de desempeño de los profesionales, se demuestra que, del 100\% de empleadores que participaron en el estudio, el $66 \%$ tienen un alto grado de satisfacción con los egresados que tienen un nivel de desempeño alto. Estos resultados coinciden con lo mencionado por García et al. (2007) en su investigación sobre la opinión de empleadores, quienes concluyen que aquellos en general consideran que el desempeño laboral es bueno.

Timarán et al. (2016) indican que una evaluación del desempeño debe realizarse basada en el perfil profesional, pues solo así se podrá definir si la persona se desempeña bien o mal en su trabajo, en relación con lo que el cargo desempeñado requiere. El resultado del desempeño, en ocasiones, puede no ser exacto a la realidad y, por lo tanto, no proporcionar información suficiente para la toma de decisiones.

Al analizar los resultados encontrados en el estudio en que existe un alto porcentaje de profesionales con un desempeño alto evaluado por los empleadores, se puede evidenciar que la formación de los profesionales está cumpliendo satisfactoriamente la misión y visión institucional. De acuerdo a los resultados obtenidos en este estudio, el $50 \%$ de los egresados laboran en instituciones del sector privado, lo cual se relaciona con lo mencionado por Martínez et al. (2018) y difiere con los estudios de Núñez y Melchor (2012), quienes perciben que el 90\% de sus egresados se encuentran ubicados laboralmente en el sector público, al igual que en el estudio realizado por Crespo-Knopfler et al. (2009).

Al analizar los resultados obtenidos en la investigación, en la que la mayoría de los empleadores indicaron que los profesionales egresados 
de la Universidad de Boyacá tienen un nivel de desempeño alto, se ve que este tipo de ejercicio es significativo, ya que muestra que los egresados responden eficazmente a las necesidades laborales de las instituciones o empresas con relación a su desarrollo personal, profesional y social. Se indica que una evaluación del desempeño debe realizarse basada en el perfil laboral, pues solo así se podrá definir si la persona desempeña bien o mal su trabajo en relación con la posición que ocupa. El resultado de la evaluación del desempeño, en algunas ocasiones, puede no estar apegado a la realidad y por lo tanto no proporcionar información suficiente para la toma de decisiones. Para obtener resultados precisos y que estos proporcionen información clara, la evaluación debe desarrollarse en función de cómo se ha definido el perfil de egreso y de las competencias que este implica.

Por otra parte, se evidencia un alto porcentaje de profesionales egresados de la Universidad de Boyacá con un desempeño alto, con lo que se puede referir que la formación de los profesionales se está cumpliendo satisfactoriamente, con relación a la misión institucional descrita así: "Inspirados en el poder del saber, formar hombres y mujeres libres, críticos y comprometidos socialmente" (Universidad de Boyacá, 2016, p. 19).

Finalmente, el enfoque por competencias tiene el potencial para convertirse en una oportunidad efectiva para el mejoramiento en la calidad de la formación. En tal sentido, representa un reto que debe ser asumido, aceptado e integrado en la cultura académica de la institución y de todos los actores involucrados en el proceso formativo. A nuestro juicio, el enfoque por competencias constituye un modelo que permite ajustar el currículo, fortalecer la integralidad y con ello superar la desarticulación entre la teoría y la práctica, lo que a su vez acorta de esta manera la distancia entre la educación universitaria y la práctica profesional con un enfoque social.

\section{Referencias Bibliográficas}

1. Artess, J., Hooley, T., \& Mellors, B. R. (2017). Employability: A review of the literature 2012-2016. Higher Education Academy. http://hdl.handle.net/10545/621285

2. Batista, C. A. \& Guacari, V. W. (2019). Efectos de la entrada en el mercado de tiendas Ara en los pequeños comerciantes de la ciudad de Cartagena. Revista Innova ITFIP, 5(1), 40-49. http://revistainnovaitfip.com/index.php/innovajournal/article/view/54

3. Burke, C., Scurry, T., Blenkinssop, J. \& Katy, G. (2017). Critical perspectives on graduate employability. En M. Tomlinson \& L. Holmes (Eds.), Graduate employability in context. Theory, research and debate (pp. 87- 107). Palgrave Macmillan.

4. Cifuentes, F. P. (2017). Las diez competencias fundamentales para la empleabilidad según egresados, profesorado y profesionales de la traducción y la interpretación. Quaderns. Revista de Traducción, 24, 197-216. https://ddd.uab.cat/pub/quaderns/quaderns_a2017n24/quaderns_a2017n24p197.pdf

5. Comisión Europea. (2004). Competencias clave para un aprendizaje a lo largo de la vida. Un marco de referencia europeo. Madrid: Dirección General de Educación y Cultura. http://www.educastur.princast.es/info/calidad/indicadores/doc/comision_europea.pdf 
6. Corredor, G. S., Aguilar, T. C., Cely, B. J., Bernal, A. A. \& Correal, C. A. (2020). Desempeño de los egresados desde la percepción de los empleadores. Cultura Educación Y Sociedad, 12(1), 105118. https://doi.org/10.17981/cultedusoc.12.1.2021.07

7. Crespo-Knopfler, S., González-Velázquez, M., Cuamatzi-Peña, M., Domínguez-Villanueva, L., \& González-Nila, L. (2018). Percepción de empleadores sobre el Licenciado en Enfermería de la Facultad de Estudios Superiores Zaragoza. Enfermería Universitaria, 6(1). https://doi.org/10.22201/eneo.23958421e.2009.1.341

8. Del Socorro, L. D. (2017). Satisfacción de empleadores por el cumplimento del encargo social de los egresados de maestrías de amplio acceso. Humanidades Médicas, 17(2), 338-353. Recuperado en 11 de marzo de 2021, de http://scielo.sld.cu/scielo.php?script=sci_arttext\&pid=S1727-81202017000200007\&lng=es\&tlng=es

9. Freire, M., Teijeiro, M., \& País, C. (2011). Políticas educativas y empleabilidad: ¿cuáles son las competencias más influyentes? Archivos Analíticos de Políticas Educativas, 19(28), 1-24. http://epaa.asu.edu/ojs/article/view/911

10. García, A. D., Sepúlveda, A. J., Arboleda, P. G., Restrepo, R. S., Garcés, G. L. \& Moreano, K. V. (2019). Estudios de graduados en educación superior: análisis relacional y comparado desde el ámbito internacional. Revista Virtual Universidad Católica del Norte, (57), 117-136. https://doi.org/10.35575/rvucn.n57a9

11. García, B. M. \& Cárdenas, S. E. (2017). La inserción laboral en la Educación Superior. La perspectiva latinoamericana. Educación XXI. (En prensa).

12. García, C., López, L., Osorio, L. \& Realpe, C. (2007). Desempeño profesional de los egresados del programa de Enfermería de la Universidad de Caldas y su relación con la Ley de Seguridad Social en Salud y con el perfil de formación (Manizales 1995-2004). Revista Hacia la Promoción de la Salud, XII(12), 91-108.

13. Garzón, C. A. (2018). Modelo para el seguimiento y acompañamiento a graduados (SAG), una visión holística de la gestión de la calidad de la educación superior. Educación, XXVII(52), 201-218. https://doi.org/10.18800/educacion.201801.011

14. Guartán, A., Torres, K. \& Ollague, J. (2019). La evaluación del desempeño laboral desde una perspectiva integral de varios factores. 593 Digital Publisher, 4(6);1-14. https://doi.org/10.33386/593dp.2019.6.139

15. Jackson, D. (2012). Business undergraduates' perceptions of their capabilities in employability skills: Implications for industry and higher education. Industry and Higher Education, 26(5), 345356. https://ro.ecu.edu.au/cgi/viewcontent.cgi?referer= https://www.google.com/\&httpsredir=1\&article=1294\&context=ecuworks2012 
16. Latiesa, M., Núñez, J., \& Martínez, R. (2001). Políticas y sociología: ámbitos académico y laboral. Universidad de Granada.

17. Lévy, L. (2003). Gestión de las competencias: cómo analizarlas, cómo evaluarlas, cómo desarro llarlas. Gestión

18. Martínez, R. J., Vergara, A. V., Quintero, J. N., Suarez, T. D., Miranda, M. C. \& Pereira, P. M. (2018). Opinión de los empleadores sobre el desempeño de egresados del programa de Enfermería de la Universidad de Sucre. Colombia, 39(29),7-15. https://www.researchgate.net/publication/326904818_Opinion_de_los_empleadores_sobre_el_desempeno_de_egresados_del_Programa_de_Enfermeria_de_la_Universidad_de_Sucre_Colombia

19. McCarthy, J. (2016). La orientación profesional y las habilidades para la gestión de la carrera. Una perspectiva internacional. En A. Manzanares y C. Sanz (Eds.), Orientación profesional. Fundamentos y estrategias (pp. 49-64). UCLM.

20. Mertens, L. (2001). La Gestión por Competencia Laboral en la Empresa y la Formación Profesional.

21. Misión de Ciencia, Educación y Desarrollo. (1994) Colombia al filo de la oportunidad. Codema.

Núñez, F. \& Melchor, A. (2012). Opinión de empleadores sobre desempeño de Egresados de la Facultad de Enfermería Poza Rica, México. ENE, Revista de Enfermería, 4(2).

http://ene-enfermeria.org/ojs/index.php/ENE/article/view/131/114

22. Orellana, N. (2018). Consideraciones sobre empleabilidad en educación superior. Calidad en la Educación, 48, 273-291.

https://scielo.conicyt.cl/pdf/caledu/n48/0718-4565-caledu-48-00273.pdf

23. Palmer, A., Montaño, J. \& Palou, M. (2009). Las competencias genéricas en la educación superior. Estudios comparativos entre la opinión de empleadores y académicos. Psicothema, 21(3), 433438. http://www.psicothema.com/psicothema.asp?id=3650

24. Peiro, J., Roig, J., González-Romá, V. y Gamboa, J. (2009). Estudio de las demandas de los empleadores de titulados universitarios de la Provincia de Valencia. Síntesis de Resultados. España: Observatorio de Inserción Profesional y Asesoramiento Laboral de la Universitat de València (OPAL). https://www.uv.es/opalweb/estudi\%20i\%20analisi/Analisi\%20demanda\%20empresari/Primer\%20estudi/Sintesi_de_Informe_General_de_Resultats.pdf

25. Rico, R. (2003). Nuevas competencias y nuevas formas de organización del trabajo. I Congreso de Inserción Laboral de los Titulados Universitarios. Valladolid: Universidad Europea Miguel de Cervantes. 
26. Rodríguez, P., Hincapié, J., Agudelo, A. \& Ramírez, R. (2012). Percepción de empleadores sobre las competencias de graduados del Programa de Enfermería de la Fundación Universitaria del Área Andina de Pereira (Colombia). Revista Cultura del Cuidado, 9(2), 22-38. https://revistas. unilibre.edu.co/index.php/cultura/article/view/1953

26. Rothwell, A. \& Rothwell, F. (2017). Graduate employability: A critical oversigth. En M. Tomlinson \& L. Holmes (Eds.), Graduate employability in context. Theory, research and debate (pp. 41-63). Palgrave Macmillan.

27. Solé, M. M., Sánchez, T. J., Arroyo, C. F. \& Argila, I. A. (2018). Los egresados universitarios y la inserción laboral: un acercamiento al panorama latinoamericano y español. Revista CEA, 4(8), 67-74. https://doi.org/10.22430/24223182.1048

28. Tardif, J. (2008). Desarrollo de un programa por competencias: de la intención a la implementación. Profesorado. Revista de Currículum y Formación del Profesorado, 12(3), 1-16. http://www.ugr.es/ recfpro/rev123ART2.pdf

29. Timarán, P. S., Hernández, A. I., Caicedo, Z. S., Hidalgo, T. A. \& Alvarado, P. J. (2016). Descubrimiento de patrones de desempeño académico con árboles de decisión en las competencias genéricas de la formación profesional. Ediciones Universidad Cooperativa de Colombia. http://dx.doi.org/10.16925/9789587600490

30. omlinson, M. (2017). Introduction: Graduate employability in context: Charting a complex, contested and multi-faceted policy and research field. En M. Tomlinson \& L. Holmes (Eds.), Graduate employability in context. Theory, research and debate (pp. 1-40). Palgrave Macmillan. Universidad de Boyacá. (2016). Competencias Generales de la Institución. Documentos Institucionales.

31. Ediciones Universidad de Boyacá.

Villa, S. \& Villa, L. (2007). El aprendizaje basado en competencias y el desarrollo de la dimensión social en las universidades. Educar, 40, 49-67. https://raco.cat/index.php/Educar/article/view/119469

32. Zarta Barrios, J. (2017). Análisis comparativo de la Gerencia de miPymes. Revista INNOVA ITFIP, 1(1), 66-71. http://revistainnovaitfip.com/index.php/innovajournal/article/view/14 